Article

\title{
Unravelling the Features of Success of VIM-Producing ST111 and ST235 Pseudomonas aeruginosa in a Greek Hospital
}

\author{
Costas C. Papagiannitsis ${ }^{1, *(1)}$, Aggeliki Verra ${ }^{1}$, Vasiliki Galani ${ }^{1}$, Stelios Xitsas ${ }^{1}$, \\ Ibrahim Bitar ${ }^{2}$, Jaroslav Hrabak ${ }^{2}$ and Efthymia Petinaki ${ }^{1}$ \\ 1 Department of Microbiology, University Hospital of Larissa, 41110 Larissa, Greece; jlkverra@yahoo.gr (A.V.); \\ galanivasiliki@gmail.com (V.G.); stexit007@gmail.com (S.X.); petinaki@uth.gr (E.P.) \\ 2 Biomedical Center, Faculty of Medicine, Charles University, 32300 Pilsen, Czech Republic; \\ ibrahimbitar5@gmail.com (I.B.); jaroslav.hrabak@lfp.cuni.cz (J.H.) \\ * Correspondence: c.papagiannitsis@gmail.com; Tel.: +30-24-1350-2532
}

Received: 4 November 2020; Accepted: 26 November 2020; Published: 28 November 2020

check for updates

\begin{abstract}
The objective of this study was to analyze the characteristics that contribute to the successful dissemination of VIM-producing Pseudomonas aeruginosa (P. aeruginosa), belonging to ST111 and ST235, in a Greek hospital. A total of 120 non-repetitive P. aeruginosa, which had meropenem minimal inhibitory concentrations (MICs) greater than $2 \mathrm{mg} / \mathrm{L}$, were studied. VIM-encoding genes were amplified and sequenced within their integrons. Isolates were typed by multilocus sequence typing (MLST). Six VIM-producers, representative of different integron structures and sequence types (STs), were completely sequenced using Illumina platform. Sixty-one $P$. aeruginosa were confirmed to produce VIM-type carbapenemases. ST111 dominated $(n=34)$ among VIM-producers, while 15 VIM-producers belonged to ST235. The bla ${ }_{\mathrm{VIM}}$-like genes were located in three integron types, including In59, In595 and In1760, which were integrated into P. aeruginosa chromosomes. Whole genome sequencing (WGS) data demonstrated that ST111 and ST235 MBL producers carried several resistance and virulence genes. Additionally, the presence of type I-C and type I-E clustered regularly interspaced short palindromic repeats (CRISPR)/Cas locus was observed in ST235 and ST395 isolates, respectively. In conclusion, our findings confirmed the clonal spread of ST111 P. aeruginosa, carrying the VIM-2-encoding integron In59, in the University Hospital of Larissa (UHL). In addition, they highlighted the important role of high-risk clones, ST111 and ST235, in the successful dissemination and establishment into hospital settings of clinically important pathogens carrying resistance determinants.
\end{abstract}

Keywords: VIM; ST235; ST111; Illumina sequencing; class 1 integrons; CRISPR/Cas system

\section{Introduction}

Pseudomonas aeruginosa is an opportunistic pathogen causing a wide range of infections, including pulmonary infections, wound and sepsis. P. aeruginosa show a great tendency to form biofilms on medical devices [1]. Additionally, P. aeruginosa express virulence factors, like exotoxins, proteases and pigment. These factors help the bacterium to facilitate successful colonization and infection across a wide range of environments.

Furthermore, P. aeruginosa show a high prevalence of antimicrobial resistance. Approximately $13 \%$ of $P$. aeruginosa, causing infections, are multidrug-resistant (MDR) strains. Therefore, recently, the World Health Organization (WHO) has considered P. aeruginosa as a target of high priority for the development of new antibiotics [2]. 
The MDR phenotype is developed mainly through over-expression of the intrinsic AmpC-type cephalosporinase, inactivation or loss of the OprD porin and/or upregulation of efflux pumps. In addition, the acquisition of resistance genes, including those encoding $\beta$-lactamases, like extended-spectrum- $\beta$-lactamases (ESBLs) or metallo- $\beta$-lactamases (MBLs) [3], contributes to the development of the MDR phenotype. MBLs are zinc-dependent enzymes hydrolyzing all $\beta$-lactams, except monobactams. Their activity is not affected by the currently available $\beta$-lactamase inhibitors. Contrary to $b l a_{\mathrm{NDM}}$ genes, $b l a_{\mathrm{IMP}}$ and bla $a_{\mathrm{VIM}}$ occur as gene cassettes in class 1 integrons [4]. These integrons often also carry genes conferring resistance to other antibiotics, like trimethoprim, aminoglycosides and chloramphenicol.

In Greece, the first outbreak of P. aeruginosa isolates harboring bla $a_{\mathrm{VIM}-1}$ took place in AHEPA University Hospital of Thessaloniki during the period of 1996-1998 [5]. In 2001, carbapenem-resistant P. aeruginosa harboring the bla $a_{\mathrm{VIM}-2}$ gene predominated among 15 Greek hospitals. In most of the cases, bla $a_{\mathrm{VIM}-2}$ was found in the variable region of class 1 integron In56 or In59 [6]. Moreover, in 2001, bla $\mathrm{VIM}-4$ was characterized from a clinical P. aeruginosa recovered in the University Hospital of Larissa (UHL) [7], central Greece. During 2001-2002, in UHL, the frequency of carbapenem-resistant P. aeruginosa reached $35 \%$, and P. aeruginosa producing VIM-2 or VIM-4 MBLs predominated [8]. According to the multilocus sequence typing (MLST) Oxford scheme, ST111 and ST235 have been the prevalent types among VIM-producing P. aeruginosa in our region [9].

The last data published from our institution referred to MBL-producing P. aeruginosa collected from central Greece, in 2011 [9]. However, it is important to continuously monitor and study the molecular epidemiology of carbapenem-resistant $P$. aeruginosa, which constantly evolves in a global level, especially in endemic areas as these regions constitute the focus for such strains. Therefore, the molecular epidemiology of MBL-producing P. aeruginosa collected in 2018 from patients treated in UHL was investigated. In addition, we aimed to analyze the characteristics that contribute to the success of epidemic clones ST111 and ST235.

\section{Materials and Methods}

\subsection{Clinical Isolates, Identification and Susceptibility Testing}

A collection of nonrepetitive 120 P. aeruginosa, isolated in 2018 from patients treated in UHL, was analyzed in this study. For each month, ten first isolates that had minimal inhibitory concentration (MIC) to meropenem greater than $2 \mathrm{mg} / \mathrm{L}$ according to 2019 EUCAST breakpoints were selected. Identification of clinical isolates was performed by the VITEK-2 system (bioMérieux, Marcy l'Étoile, France). Antimicrobial susceptibility of bacterial isolates against several antibiotics including piperacillin, piperacillin-tazobactam, cefotaxime, ceftazidime, cefepime, aztreonam, imipenem, meropenem, gentamicin, amikacin, tobramycin, chloramphenicol, tetracycline, trimethoprim-sulfamethoxazole, ciprofloxacin, and levofloxacin was also performed by the VITEK-2 system. The MICs of colistin were determined by the broth dilution method [10]. P. aeruginosa ATCC 27853 and Escherichia coli ATCC25922 were used as control strains [11]. Susceptibility data were interpreted according to the criteria (version 9.0) of the European Committee on Antimicrobial Susceptibility Testing (EUCAST) (www.eucast.org).

\subsection{Confirmation of Carbapenemase Production}

All isolates were subjected to phenotypic detection of MBLs using DDST with EDTA [12]. Genes encoding KPC, NDM, VIM, IMP and OXA-48-like carbapenemases were detected by PCR amplification [13].

\subsection{Multilocus Sequence Typing}

All P. aeruginosa isolates were analyzed by multilocus sequence typing (MLST) [14]. The database at https://pubmlst.org/paeruginosa was employed for determining STs. 


\subsection{Integron Analysis}

Variable regions of class 1 integrons with $b l a_{\mathrm{VIM}}$-like genes were amplified in two parts, from the $5^{\prime}$ conserved segment ( $\left.5^{\prime} \mathrm{CS}\right)$ to bla $a_{\mathrm{VIM}}$ cassette, and from bla $a_{\mathrm{VIM}}$ cassette to the $3^{\prime}$ conserved segment ( $\left.3^{\prime} \mathrm{CS}\right)$ [13]. VIM-encoding integrons were sequenced using an ABI 3500 sequencer (Applied Biosystems, Foster City, CA, USA). Integron sequences were analyzed using the Integrall integron database (http://integrall.bio.ua.pt) [15].

\subsection{Plasmid Analysis}

Six VIM-producing P. aeruginosa (VPP) were selected as representatives of all different STs and integron types in order to define the genetic units harboring the $b l_{\mathrm{VIM}}$-like genes. The plasmid contents of the selected isolates were analyzed by pulsed-field gel electrophoresis (PFGE) of total DNA digested with S1 nuclease (Promega, Madison, WI, USA) [16], followed by bla $a_{\mathrm{VIM}}$ hybridization [13].

\subsection{Whole Genome Sequencing}

The 6 VPP isolates, used in "plasmid analysis' experiments" were further analyzed with whole genome sequencing (WGS). For comparison purposes, nine non-carbapenemase-producing P. aeruginosa were also included. The genomic DNAs of $P$. aeruginosa were extracted using the DNA-Sorb-B kit (Sacace Biotechnologies S.r.l., Como, Italy). Multiplexed DNA libraries were prepared, using the Nextera XT Library Preparation Kit (Illumina Inc., San Diego, CA, USA), and 300-bp paired-end sequencing was performed on Illumina MiSeq platform (Illumina Inc., San Diego, CA, USA) using the MiSeq v3 600-cycle Reagent Kit (Illumina Inc., San Diego, CA, USA). Initial paired-end reads were quality trimmed using Trimmomatic tool v0.32. Then, reads were assembled via de Bruijn graph-based de novo assembler SPAdes v3.14.0. The sequence gaps were confirmed by PCR-based strategy and Sanger sequencing of the amplicons. For sequence analysis and annotation, the BLAST algorithm (www.ncbi.nlm.nih.gov/BLAST), the open reading frame (ORF) finder tool (www.bioinformatics.org/ $\mathrm{sms} /$ ) and the ISFinder database (www-is.biotoul.fr/) were employed. Comparative genome alignment was conducted using Mauve v.2.3.1. (http://darlinglab.org/mauve/mauve.html).

WGS data were analyzed using the ResFinder 3.2 tool (https://cge.cbs.dtu.dk/services/ResFinder/), with an identity threshold $>90 \%$ [17], and with the Virulence Factors Database (VFDB; http://www. mgc.ac.cn/VFs/main.htm) [18]. Finally, the CRISPRCasFinder program (https://crisprcas.i2bc.parissaclay.fr/CrisprCasFinder/Index) was utilized for the easy detection of clustered regularly interspaced short palindromic repeats (CRISPR) and Cas-associated genes [19].

\subsection{Nucleotide Sequence Accession Numbers}

Nucleotide sequences representing the genetic context of VIM-encoding integrons In595 and In1760 were submitted to the GenBank under accession numbers MT428323 and MT437279, respectively.

\section{Results}

Twenty-four P. aeruginosa isolates had MIC to meropenem of 4-8 $\mathrm{mg} / \mathrm{L}$ and were categorized as susceptible at increased exposure, whereas 96 isolates had MIC $>8 \mathrm{mg} / \mathrm{L}$ and were categorized as resistant according to the 2019 EUCAST definition. The majority of P. aeruginosa isolates were resistant to cefepime $(n=87,72.5 \%)$, ceftazidime $(n=93,77.5 \%)$, imipenem $(n=96,80 \%)$, piperacillin-tazobactam $(n=109,90.8 \%)$ and piperacillin $(n=114,95.0 \%)$. Only $62(51.7 \%)$ isolates were resistant to aztreonam. One-hundred eight (90.0\%) of P. aeruginosa isolates also exhibited resistance to levofloxacin, $104(86.7 \%)$ were resistant to ciprofloxacin, $92(76.7 \%)$ to tobramycin, $78(65.0 \%)$ to amikacin, and $70(58.3 \%)$ to gentamicin.

In 70 P. aeruginosa isolates, the EDTA-meropenem test was positive, indicating MBL production. However, 61 out of 70 the isolates, being positive in the EDTA-meropenem test, were positive for bla genes. Other types of carbapenemase-encoding genes were not detected. 
The population structure of P. aeruginosa isolates studied by MLST showed that the VPP isolates included four sequence types (STs) (Figure 1). ST111 was the most predominant comprising 34 VIM-producing isolates (Table 1). Twenty five VIM producers were distributed in STs $235(n=15)$ and $395(n=10)$. STs 111, 235 and 395 have been assumed as "high-risk clones" [20]. The remaining VIM-producing P. aeruginosa isolates belonged to ST773 $(n=2)$. However, higher genetic diversity was observed in the group of non-carbapenemase-producing isolates (Table 1$)$. This group of isolates $(n=59)$ comprised 11 clones, with STs $235(n=14)$ and $111(n=11)$ accounting for 25 isolates.

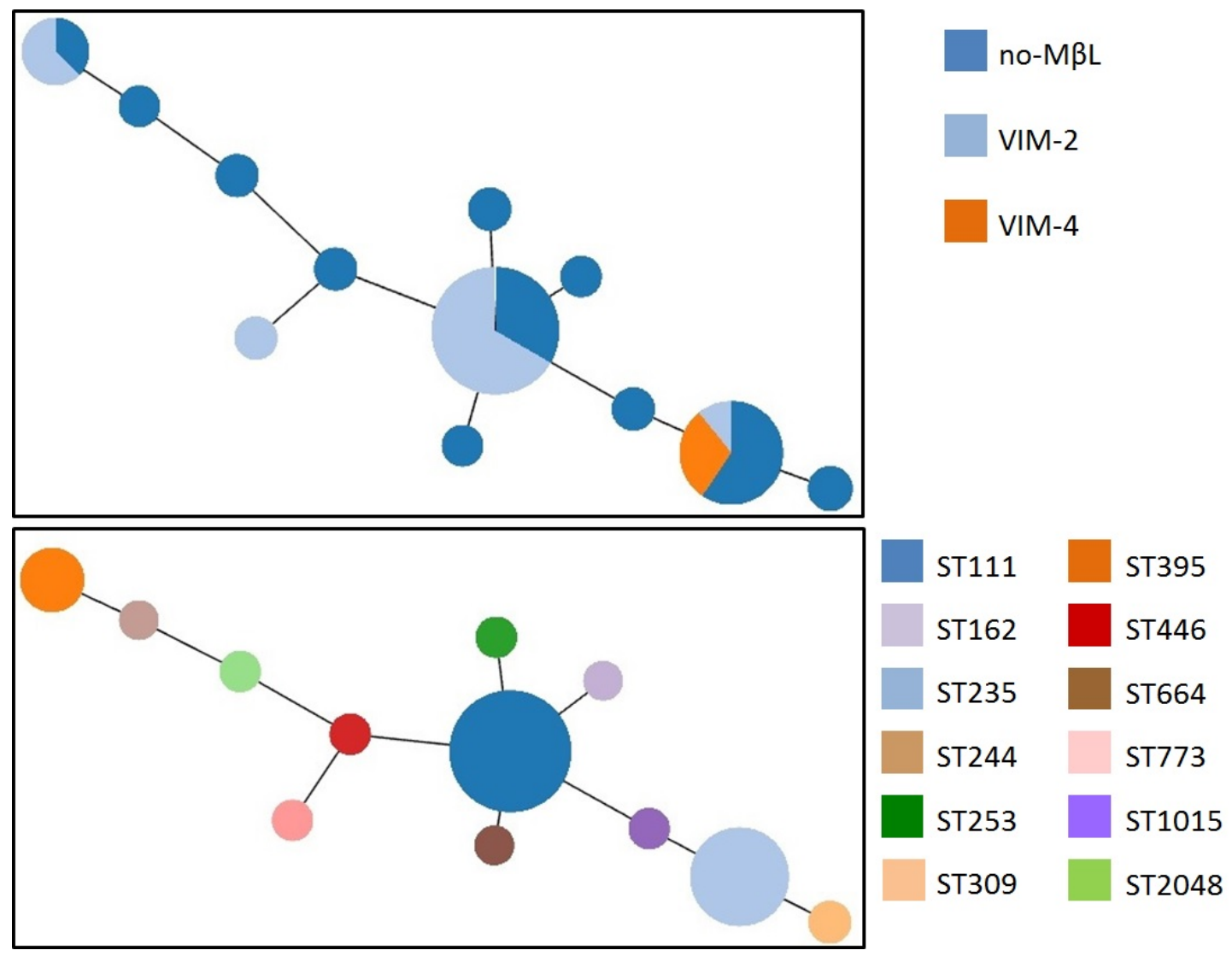

Figure 1. Minimal spanning tree of 120 Pseudomonas aeruginosa isolates, recovered from University Hospital of Larissa (UHL) during 2018, showing sequence types (STs) and carbapenemase content. Each circle corresponds to an ST. The area of each circle is proportional to the number of isolates.

Characterization of the regions carrying the carbapenemase-encoding genes revealed that bla $a_{\mathrm{VIM}}$-like genes were located in three main types of class 1 integrons (Table 1 ). The most common integron was In59 identified in 40 P. aeruginosa, including 34 ST111, 4 ST235 and 2 ST773 isolates. In59 possessed $a a c A 29 a, b l a_{\mathrm{VIM}-2}$ and $a a c A 29 b$ gene cassettes [21]. However, the 11 remaining ST235 VIM producers carried the class 1 integron In595 [22], including an array of bla $a_{\mathrm{VIM}-4}$, arr-7, aacA4, bla PSE-1 $_{1}$ cassettes. In595 has been previously described in two MBL-producing isolates imported from Greece and Cyprus in Scandinavia. One of those P. aeruginosa isolates was also ST235. Finally, among ST395 VIM producers, the class 1 integron In1760, whose variable region comprised the gcu205, bla OXA-10, $a a c A 4, b l a_{\mathrm{VIM}-2}$, and smr-2 cassettes, was identified. The gcu 205 cassette encoded a hypothetical protein of unknown function. The $b l a_{\mathrm{OXA}-10}$ and $b l a_{\mathrm{VIM}-2}$ genes mediated resistance to $\beta$-lactams, while the aac $A 4$ cassette conferred to aminoglycosides. The last cassette, smr-2, encoded a small multi-drug resistance protein mediating efflux of lipophilic cations. The majority $(n=57)$ of VIM-producing isolates were resistant to meropenem. However, four VIM-2 producers (three ST111 and one ST395 isolates) exhibited meropenem MIC 4-8 mg/L and were categorized as susceptible at increased exposure. 
Table 1. Population structure of 120 P. aeruginosa isolates, recovered from UHL during 2018.

\begin{tabular}{cccc}
\hline ST & MBL & No. of Isolates & Integrons with bla VIM Genes \\
\hline ST111 & VIM-2 & 34 & In59 \\
& - & 17 & - \\
ST162 & - & 1 & - \\
ST235 & VIM-2 & 4 & In59 \\
& VIM-4 & 11 & In595 \\
& - & 22 & - \\
ST244 & - & 1 & - \\
ST253 & - & 2 & - \\
ST309 & - & 3 & - \\
ST395 & VIM-2 & 10 & In1760 \\
& - & 6 & - \\
ST446 & - & 2 & - \\
ST664 & - & 1 & In59 \\
ST773 & VIM-2 & 2 & - \\
ST1015 & - & 2 & - \\
ST2048 & - & 2 & \\
\hline
\end{tabular}

S1 profiling did not observer any DNA bands that could be assigned to plasmids, and the $b l a_{\mathrm{VIM}}$-like probe hybridized with only the largest DNA bands, indicating the chromosomal location of the VIM-encoding integrons. Thus, the genomes of these isolates (Table 2) were sequenced by the Illumina platform. Sequence analysis showed that, in all cases, VIM-encoding integrons were inserted into $P$. aeruginosa chromosomes.

The In59-like integron was located in a Tn5060-like transposon integrated into the chromosome of ST111 P. aeruginosa isolates. However, insertion of the Tn3-like transposon Tn4661 [23] probably deleted the Tn5060 mer module. Tn4661 was found next to a partially deleted ICE1-like integrative conjugative element $(\triangle \mathrm{ICE} 1)$, which was inserted into the tRNALys gene (PA4541.1 in GenBank accession no. AE004091). A similar structure has been observed in VIM-producing P. aeruginosa isolates belonging to ST111 isolated in Czech hospitals [13].

In595 integron was in integrative conjugative element ICEPae3483, identified in the P. aeruginosa chromosome (Figure 2). ICEPae3483 was previously found in P. aeruginosa HSV3483 (GenBank accession no. MF168944) from Portugal. The In595 was embedded in a Tn1721-like transposon. The IRi of In595 was found in tnpM of the Tn1721-like, while the 3'CS of In595 was disrupted after the start codon of orf5 by an IS6100 element.

In ST395 VIM-2 producers, In1760 was associated with a composite transposon, bounded by two copies of the ISPsp 7 insertion sequence in opposite orientation. The bla $a_{\mathrm{VIM}-2}$-carrying transposon was inserted in a novel genomic island (GI) (Figure 2), designated PAGI-8709. PAGI-8709 included open reading frames (ORFs) encoding hypothetical proteins of unknown function and proteins of various functions (e.g., regulation of gene expression, DNA recombination and metabolic activities). GIs closely related to PAGI-8709 have not been found previously in P. aeruginosa. PAGI-8709 was integrated in P. aeruginosa chromosome into gene encoding for tRNALys (PA0729 in GenBank accession no. AE004091). The $5^{\prime} \mathrm{CS}$ of the integron was intact, with the IRi of the integron being next to a 9685-bp segment (nucleotide 163493 to 166551 in GenBank accession no. MT437279), presenting 100\% identity with $\alpha$ Tn3-like sequence from Pseudomonas sp. strain AG1 (GenBank accession no. CP045739), whereas an ISPa17 element was inserted into the $3^{\prime} \mathrm{CS}$ of the integron, downstream of orf5.

Additional genes conferring resistance to aminoglycosides, $\beta$-lactams and chloramphenicol were identified in the majority of the sequenced isolates (Table 2), as indicated by analysis of WGS data by the ResFinder 3.2 tool. Additionally, WGS data confirmed the absence of known carbapenemase-encoding genes in nine $P$. aeruginosa isolates being positive in the EDTA-meropenem test, but negative in PCR screening. 
Table 2. Characteristics of 18 P. aeruginosa characterized by Illumina sequencing.

\begin{tabular}{|c|c|c|c|c|c|c|}
\hline Isolate & ST & $\begin{array}{c}\text { MBL } \\
\text { Content }\end{array}$ & $\begin{array}{c}\text { Integrons } \\
\text { with bla } \\
\text { Genes }\end{array}$ & Additional Resistance Genes & Virulence Genes & $\begin{array}{l}\text { CRISPR/Cas } \\
\text { System }^{\text {a }}\end{array}$ \\
\hline Pae8397-Lar & 111 & VIM-2 & In59 & $b l a_{\mathrm{PAO}}, b l a_{\mathrm{OXA}-395}, a p h\left(3^{\prime}\right)-I I b, c a t B 7$ & $\begin{array}{c}\text { algD, aprA, lasB, plcH, plcN, exoS, exoT, exoY, } \\
\text { phzM, phzS, phzH, pilA, pilB, toxA }\end{array}$ & - \\
\hline Pae9981-Lar & 111 & VIM-2 & In59 & $b l a_{\mathrm{PAO}}, b l a_{\mathrm{OXA}-395}, a p h\left(3^{\prime}\right)-I I b, c a t B 7$ & $\begin{array}{c}\text { algD, apr } A, \text { las } B, \text { plcH, plcN, exoS, exoT, exoY, } \\
\text { phzM, phzS, phzH, pilA, pilB, toxA }\end{array}$ & - \\
\hline Pae8709-Lar & 235 & VIM-4 & In595 & $b l a_{\mathrm{PAO}}, b l a_{\mathrm{OXA}-35}, b l a_{\mathrm{OXA}-488}, a p h\left(3^{\prime}\right)-I I b, c a t B 7$ & $\begin{array}{c}\text { algD, apr } A, \operatorname{las} B, \text { plcH, plcN, exoT, exoU, exoY, } \\
\text { phzM, phzS, phzH, pilB, toxA }\end{array}$ & Type I-C \\
\hline Pae9938-Lar & 235 & VIM-4 & In595 & $b l a_{\mathrm{PAO}}, b l a_{\mathrm{OXA}-35}, b l a_{\mathrm{OXA}-488}, a p h\left(3^{\prime}\right)-I I b, c a t B 7$ & $\begin{array}{c}\text { algD, apr } A, \operatorname{las} B, \text { plcH, plcN, exoT, exoU, exoY, } \\
\text { phzM, phzS, phzH, pilB, tox } A\end{array}$ & Type I-C \\
\hline Pae8414-Lar & 395 & VIM-2 & In1760 & $\begin{array}{c}b l a_{\mathrm{PAO}}, b l a_{\mathrm{OXA}-488}, a a d A 6, a p h\left(3^{\prime}\right)-I I b, \text { aph }\left(3^{\prime}\right)-V i a, \\
\text { catB7 }\end{array}$ & $\begin{array}{c}\text { algD, aprA, lasB, plcH, plcN, exoS, exoT, exoY, } \\
\text { phzM, phzS, phzH, pilB, tox } A\end{array}$ & Type I-E \\
\hline Pae9047-Lar & 395 & VIM-2 & In1760 & $\begin{array}{c}\text { bla }_{\mathrm{PAO}}, b l a_{\mathrm{OXA}-488}, a_{\text {adA6, }} \text { aph }\left(3^{\prime}\right)-I I b, \text { aph }\left(3^{\prime}\right)-V i a, \\
\text { catB7 }\end{array}$ & $\begin{array}{c}\text { algD, aprA, lasB, plcH, plcN, exoS, exoT, exoY, } \\
\text { phzM, phzS, phzH, pilA, pilB, toxA }\end{array}$ & Type I-E \\
\hline Pae112-Lar & 162 & No & - & $b l a_{\mathrm{PAO}}, b l a_{\mathrm{OXA}-494}, a a c A 29, \operatorname{aph}\left(3^{\prime}\right)-I I b$, catB7, sul1 & $\begin{array}{c}\text { algD, aprA, lasB, plcH, plcN, exoT, exoU, exoY, } \\
\text { phzM, phzS, phzH, pilB, toxA }\end{array}$ & - \\
\hline Pae69-Lar & 235 & No & - & $\begin{array}{l}\text { bla } a_{\mathrm{PAO}}, b l a_{\mathrm{OXA}-2}, b l a_{\mathrm{OX}}-488, b l a_{\mathrm{PER}-1}, a a c A 4, \\
\text { aadA2, ant }\left(2^{\prime \prime}\right)-I a, \operatorname{aph}\left(3^{\prime}\right)-I I b, \operatorname{str} A, \operatorname{str} B, \operatorname{sul1}\end{array}$ & $\begin{array}{c}\text { algD, aprA, lasB, plcH, plcN, exoT, exoU, exoY, } \\
\text { phzM, phzS, phzH, pilB, toxA }\end{array}$ & - \\
\hline Pae90-Lar & 235 & No & - & $\begin{array}{c}\text { bla }_{\mathrm{PAO}}, b l a_{\mathrm{OXA}-35}, b l a_{\mathrm{OXA}-488}, a a c A 4, a p h\left(3^{\prime}\right)-I I b, \\
\text { catB7, sul1 }\end{array}$ & $\begin{array}{c}\text { algD, aprA, las } B, p l c H, p l c N, \text { exoT, exoU, exoY, } \\
\text { phzM, phzS, phzH, pilB, toxA }\end{array}$ & Type I-C \\
\hline Pae91-Lar & 244 & No & - & $b l a_{\mathrm{PAO}}, b l a_{\mathrm{OXA}-494}, a p h\left(3^{\prime}\right)-I I b, c a t B 7$ & $\begin{array}{c}\operatorname{alg} D, \operatorname{apr} A, \operatorname{las} B, \text { plcH, plcN, exoT, exoU, exoY, } \\
\text { phzM, phzS, phzH, pilB, toxA }\end{array}$ & Type I-F \\
\hline Pae462-Lar & 253 & No & - & $b l a_{\mathrm{PAO}}, b l a_{\mathrm{OXA}-488}, a p h\left(3^{\prime}\right)-I I b, c a t B 7$ & $\begin{array}{c}\text { algD, aprA, lasB, plcH, plcN, exoT, exoU, exoY, } \\
\text { phzM, phzS, phzH, pilB, toxA }\end{array}$ & Type I-F \\
\hline Pae100-Lar & 299 & No & - & $b l a_{\mathrm{PAO}}, b l a_{\mathrm{OXA}-494}, a p h\left(3^{\prime}\right)-I I b$, catB7 & $\begin{array}{c}\text { algD, aprA, lasB, plcH, plcN, exoS, exoT, exoY, } \\
\text { phzM, phzS, phzH, pilB, toxA }\end{array}$ & - \\
\hline Pae86-Lar & 446 & No & - & $b l a_{\mathrm{PAO}}, b l a_{\mathrm{OXA}-395}, a p h\left(3^{\prime}\right)-I I b, c a t B 7$ & $\begin{array}{c}\text { algD, aprA, lasB, plcH, plcN, exoT, exoU, exoY, } \\
\text { phzM, phzS, phzH, pilA, pilB, toxA }\end{array}$ & - \\
\hline Pae64-Lar & 664 & No & - & $b l a_{\mathrm{PAO}}, b l a_{\mathrm{OXA}-50}, a p h\left(3^{\prime}\right)-I I b, c a t B 7$ & $\begin{array}{c}\text { algD, aprA, lasB, plcH, plcN, exoS, exoT, exoY, } \\
\text { phzM, phzS, phzH, pilB, toxA }\end{array}$ & - \\
\hline Pae475-Lar & 2048 & No & - & $b l a_{\mathrm{PAO}}, b l a_{\mathrm{OXA}-494}, a p h\left(3^{\prime}\right)-I I b, c a t B 7$ & $\begin{array}{c}\text { algD, apr } A, \text { lasB, plcH, plcN, exoS, exoT, exoY, } \\
\text { phzM, phzS, phzH, pilA, pilB, toxA }\end{array}$ & - \\
\hline
\end{tabular}

a The absence of a CRISPR/Cas system is indicated by a ' - '. 

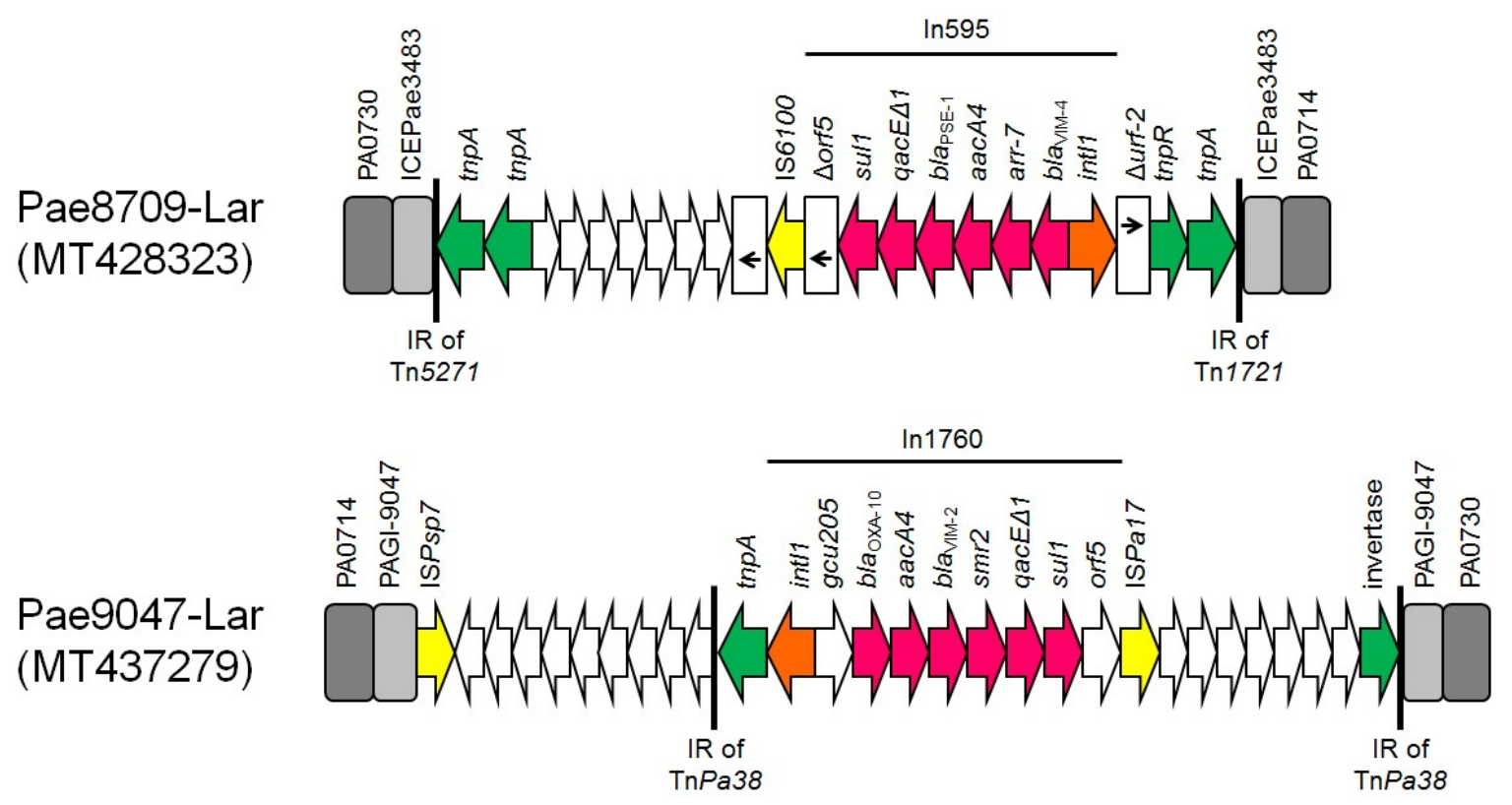

Figure 2. Linear maps of the genetic context of VIM-encoding class 1 integrons In595 and In1760 integrated into the P. aeruginosa chromosome. Arrows show the direction of transcription of open reading frames (ORFs), while truncated ORFs appear as rectangles (arrows within rectangles indicate the direction of transcription). Resistance genes, insertion sequences (IS), and transposases are shown in pink, yellow, and green, respectively. intI1 genes are shaded orange. Sequences associated with genomic islands (GIs) are shaded light gray; dark gray rectangles indicate the P. aeruginosa chromosome. The remaining genes are shown in white.

Examination of quinolone resistance-determining regions of $g y r A, g y r B$, parC, and parE and of the mexR, $n f x B$, and mexT genes, which regulate the MexAB-OprM, MexCD-OprJ, and MexEF-OprN multidrug efflux systems [24], revealed the presence of several point mutations predicted to result in several amino acid substitutions (Table 3). The majority of these amino acid substitutions have been identified previously from both ciprofloxacin-susceptible and ciprofloxacin-resistant $P$. aeruginosa isolates. However, in GyrA, the T83I amino acid substitution that previously has been associated with increased quinolone resistance [25] was found in all ciprofloxacin-resistant isolates. Furthermore, in the opr $D$ gene, point mutations predicted to result in early termination of translation were identified in six P. aeruginosa isolates (Table 3). This finding is in agreement with increased resistance to carbapenems [26], even in non-carbapenemase-producing isolates.

Analysis of WGS data by VFDB showed that all sequenced isolates carried several virulence genes (Table 2) that can be involved in colonization and cause bloodstream invasion, extensive tissue damage and dissemination [27]. These included secreted toxins, like exoenzyme S, exoenzyme T, exoenzyme $\mathrm{U}$ and exoenzyme $\mathrm{Y}$, subverting host cell defense and signaling systems [28]. Our results confirmed that the clone ST111 correlated with the copresence of exoS, exoT, and exoY genes, whose expression correlates with a higher risk of mortality [29]. On the other hand, clone ST235 correlated with the copresence of exoT, exoU, and exoY. In particular, ExoU is associated with lung damage and acute cytotoxicity [30]. Additionally, sequenced isolates carried genes encoding phospholipases ( $\mathrm{PlcH}$ and $\mathrm{PlcN}$ ), elastase B, alkaline protease A, exotoxin A and phenazines (Table 2), which have been previously associated with increased virulence of $P$. aeruginosa isolates [31,32].

Finally, the presence of the CRISPR/Cas system in P. aeruginosa isolates was examined. CRISPR/Cas systems provide bacteria with an adaptive immunity system against invading genetic elements [33], like bacteriophages and plasmids. A CRISPR/Cas system was found in seven of the sequenced P. aeruginosa. Isolates belonging to STs 235 and 395 exhibited type I-C and type I-E CRISPR/cas locus, respectively, while a type I-F CRISPR/Cas locus was found in ST244 and ST253 isolates (Table 2). 
Table 3. Susceptibility levels of carbapenems and ciprofloxacin, and amino acid substitutions in the GyrA, GyrB, ParC, ParE, MexR, NfxB, MexT and OprD amino acid sequences of 18 P. aeruginosa characterized by Illumina sequencing.

\begin{tabular}{|c|c|c|c|c|c|c|c|c|c|c|c|c|c|}
\hline \multirow{2}{*}{ Isolate } & \multirow{2}{*}{ ST } & \multirow{2}{*}{$\begin{array}{c}\text { MBL } \\
\text { Content }\end{array}$} & \multicolumn{3}{|c|}{$\operatorname{MIC}(\mu \mathrm{g} / \mathrm{mL})^{1}$} & \multicolumn{7}{|c|}{ Amino Acid Changes ${ }^{2}$} & \multirow[b]{2}{*}{ OprD $^{5}$} \\
\hline & & & Imp & Mem & Cip & GyrA $^{3}$ & GyrB $^{4}$ & ParC & ParE & MexR & NfxB & MexT & \\
\hline Pae8397-Lar & 111 & VIM-2 & $>16$ & $>16$ & $>4$ & $\begin{array}{l}\text { T83I, V671I, G860S, D893E, A900G, } \\
\text { S903A, + }{ }^{+} 9912,+{ }^{+} \text {E913 }\end{array}$ & SB1 & S87L, F254V, A346Q & T89I, I91T & V126E & E124A & L26V & $* 94$ \\
\hline Pae9981-Lar & 111 & VIM-2 & $>16$ & $>16$ & $>4$ & $\begin{array}{l}\text { T83I, V671I, G860S, D893E, A900G, } \\
\text { S903A, }{ }^{+} \text {S912, }{ }^{+} \text {E913 }\end{array}$ & SB1 & S87L, F254V, A346Q & T89I, I91T & V126E & E124A & L26V & $\operatorname{Dis}_{337}$ \\
\hline Pae8709-Lar & 235 & VIM-4 & $>16$ & $>16$ & $>4$ & T83I & $\begin{array}{c}\text { SB1, } \\
\text { E469D }\end{array}$ & S87L, F254V, A346Q & T89I, I91T, D533E & V126E & E124A & L26V & $* 94$ \\
\hline $\begin{array}{l}\text { Pae9938-Lar } \\
\text { Pae8414-Lar }\end{array}$ & $\begin{array}{l}235 \\
395\end{array}$ & $\begin{array}{l}\text { VIM-4 } \\
\text { VIM-2 }\end{array}$ & $\begin{array}{l}>16 \\
>16\end{array}$ & $\begin{array}{l}>16 \\
>16\end{array}$ & $\begin{array}{l}>4 \\
>4\end{array}$ & $\begin{array}{c}\text { T83I } \\
\text { T83I + E909 +S910 }\end{array}$ & SB1 & $\begin{array}{l}\text { S87L, F254V, A346Q } \\
\text { S87L.F254V,A346O }\end{array}$ & $\begin{array}{l}\text { T89I, I91T, D533E } \\
\text { T89I I91T V200M }\end{array}$ & V126E & E124A & L26V & $\begin{array}{c}\text { Non-SC } \\
* 229\end{array}$ \\
\hline & $\begin{array}{l}395 \\
395\end{array}$ & $\begin{array}{l}\text { VIM-2 } \\
\text { VIM-2 }\end{array}$ & $>16$ & $\begin{array}{l}>16 \\
>16\end{array}$ & $>4$ & $\begin{array}{l}{\mathrm{T} 83 \mathrm{I},{ }^{+} \mathrm{E} 909,+}^{+} \mathrm{S} 910 \\
\mathrm{~T} 83 \mathrm{I}+{ }^{+} \mathrm{E} 909{ }^{+} \mathrm{+} 910\end{array}$ & SB1 & & $\begin{array}{l}\text { T89I, I91T, V200M } \\
\text { T89I I91T, V200M }\end{array}$ & - & $\begin{array}{l}\text { E124A } \\
\text { F124A }\end{array}$ & $\begin{array}{l}\mathrm{L} 26 \mathrm{~V} \\
\mathrm{~L} 26 \mathrm{~V}\end{array}$ & ${ }^{*} 229$ \\
\hline $\begin{array}{l}\text { Pae9047-Lar } \\
\text { Pae112-Lar }\end{array}$ & 162 & $\begin{array}{l}\text { Noll-2 } \\
\text { No }\end{array}$ & $>16$ & $\begin{array}{l}>16 \\
>16\end{array}$ & 1 & $\begin{array}{c}\text { 1831, }{ }^{\top} \mathrm{E} 909,{ }^{\top} \mathrm{S} 910 \\
\text { T83I }\end{array}$ & $\begin{array}{l}\text { SB1 } \\
\text { SB1 }\end{array}$ & $\begin{array}{l}\text { S87L, F254V, A346Q } \\
\text { F254V, A346Q }\end{array}$ & $\begin{array}{c}\text { T89I, 191T, V200M } \\
\text { T89I, I91T, A473V, D533E }\end{array}$ & - & $\begin{array}{l}\text { E124A } \\
\text { E124A }\end{array}$ & $\begin{array}{l}\mathrm{L} 26 \mathrm{~V} \\
\mathrm{~L} 26 \mathrm{~V}\end{array}$ & $* 219$ \\
\hline Pae69-Lar & 235 & No & $>16$ & $>16$ & $>4$ & T83I & $\begin{array}{l}\text { SB1, } \\
\text { P750S }\end{array}$ & S87L, F254V, A346Q & T89I, I91T, D533E & V126E & E124A & L26V & $\mathrm{FR}_{501}$ \\
\hline Pae90-Lar & 235 & No & $>16$ & $>16$ & $>4$ & T83I & SB1 & S87L, F254V, A346Q & T89I, I91T, D533E & V126E & E124A & L26V & ${ }^{*} 142$ \\
\hline Pae91-Lar & 244 & No & $>16$ & 4 & $\leq 0.25$ & & SB1 & F254V, A346Q & T89I, I91T & & E124A & L26V & Non-SC \\
\hline Pae462-Lar & 253 & No & $>16$ & $>16$ & 1 & ${ }^{+} \mathrm{E} 909,+{ }^{+} \mathrm{S} 910$ & SB1 & F254V, A346Q & T89I, I91T, A473V, D533E & $\begin{array}{l}\text { G91E, } \\
\text { V126E }\end{array}$ & $\begin{array}{l}\text { R21H, D56G, } \\
\text { E124A }\end{array}$ & $\mathrm{L} 26 \mathrm{~V}$ & $\mathrm{FR}_{501}$ \\
\hline Pae100-Lar & 299 & No & $>16$ & 8 & $\leq 0.25$ & & SB1 & F254V, A346Q, V646L & T89I, I91T, D533E & & E124A & L26V & Non-SC \\
\hline Pae86-Lar & 446 & No & $>16$ & 4 & 2 & ${ }^{+} \mathrm{E} 909,+{ }^{+} \mathrm{S} 910$ & SB1 & F254V, A346Q, A587T & T89I, I91T, D533E & V126E & E124A & L26V & Non-SC \\
\hline Pae64-Lar & 664 & No & 2 & 12 & 1 & & SB1 & $\mathrm{F} 254 \mathrm{~V}, \mathrm{H} 262 \mathrm{Q}, \mathrm{A} 346 \mathrm{Q}$ & T89I, I91T, E215Q & $* 94$ & E124A & L26V & Non-SC \\
\hline Pae475-Lar & 2048 & No & $>16$ & 4 & $\leq 0.25$ & - & SB1 & $\mathrm{F} 254 \mathrm{~V}, \mathrm{~A} 346 \mathrm{Q}$ & T89I, I91T & - & R82L, E124A & L26V & ${ }^{*} 424$ \\
\hline
\end{tabular}

${ }^{1}$ Imp, imipenem; Mem, meropenem; Cip, ciprofloxacin. ${ }^{2}$ Sequences were compared to those under GenBank accession no. L29417 for GyrA, AB005881 for GyrB, AB003428 for ParC, AB003429 for ParE, U23763 for MexR, X65646 for NfxB, AJ007825 for MexT, and AE004091 for OprD. ${ }^{3}$ The duplication of the specific amino acids is shown by.$+{ }^{4}$ SB1 indicates substitution of amino acids G151-S152-A153-V154-P155-T156-A157-R158-S159-G160-R161-R162 to V151-P152-O153-F154-P155-L156-R157-E158-V159-G160-E161. 5 The coding sequences for OprD were analyzed for the presence of stop codons. Non-SC symbolizes the absence of a stop codon in the deduced protein sequences. ${ }^{*}$ shows the stop codon position in the deduced protein sequences. Dis symbolizes disruption of oprD locus, while FR shows the presence of a frameshift resulting in a longer OprD protein. Mutations that have been described previously to confer resistance to carbapenems are indicated in bold. 
Both ST395 isolates exhibited identical CRISPR/Cas systems, further confirming their clonal relationship. The type I-E CRISPR/Cas locus consisted of eight genes consecutively encoding the Cas2, Cas1, Cas6, Cas5, Cas7, Cse2, Cse1 and Cas3 proteins. The locus enclosed two arrays of 12 (CRISPR1) and 8 spacers (CRISPR2). The spacers, presenting variable sequences, had a common length of 32 bp. Each spacer was flanked by two direct repeats (DRs), being 29 bp long. The DRs in CRISPR1 had the 5'-CGGTTCATCCCCACGCATGTGGGGAACAC-3' consensus sequence, whereas the DRs in CRISPR2 had the 5'-CGGTTCATCCCCACACCCGTGGGGAACAC-3' consensus sequence.

The type I-C CRISPR/Cas locus consisted of seven genes consecutively encoding the Cas3, Cas5, Cas8, Cas7, Cas4, Cas1, and Cas2 proteins. The locus was followed by an array of 20 spacers, whose variable sequences were 33-36 bp long. DRs flanking the spacers had the 5'-GTCGCGCCCCGCACGGG CGCGTGGATTGAAAC-3' consensus sequence and a length of $32 \mathrm{bp}$. All ST235 isolates producing VIM-4 carbapenemase exhibited identical CRISPR/Cas systems. However, the type I-C CRISPR/Cas locus of the ST235 non-carbapenemase-producing isolate, Pae90-Lar, differed by an internal deletion erasing a spacer. This finding may indicate diverse evolution dynamics into those isolates. Type I-C locus was recently identified in ST235 isolates during a study analyzing the phylogenetic distribution of CRISPR/Cas systems in antibiotic-resistant P. aeruginosa [34].

In the ST244 isolate, Pae91-Lar, the type I-F CRISPR/Cas locus included six genes expressing the Cas1 endonuclease, Cas3/Cas2 helicase/RNAse, three Csy proteins (Csy1, Csy2, Csy3) and Cas6 endoribonuclease. The type I-F locus enclosed two arrays of 5 (CRISPR1) and 39 (CRISPR2) spacers. The spacers, presenting variable sequences, had a common length of $32 \mathrm{bp}$. Each spacer was flanked by two DRs 28 bp in length. The consensus sequence of the DRs found in CRIPSR1 was 5'-TTTTCTTAGCTGCCTATACGGCAGTGAAC-3' , whereas in CRISPR2, it was 5'-GTTCACTGCCGTG TAGGCAGCTAAGAAA-3'. Although a type I-F CRISPR/Cas locus was found in the ST253 isolate, Pae462-Lar, the locus exhibited 96.99\% similarity to that identified in Pae91-Lar. Unlike Pae91-Lar, the locus included two arrays of 13 and 18 spacers. Type I-F locus was found previously in $38.5 \%$ of clinical P. aeruginosa isolates recovered from two Brazilian hospitals [35].

A BLASTn search with the identified spacers matched P. aeruginosa chromosomal sequences as well as different mobile elements, including bacteriophages and plasmids.

\section{Discussion}

In the current study, we analyzed the molecular characteristics of $P$. aeruginosa isolates belonging to the I and R susceptibility category according to meropenem MICs, recovered during surveillance program in UHL in 2018. A total of 50.8\% of the isolates was confirmed to produce VIM-type carbapenemases. The majority $(80.3 \%)$ of carbapenemase-producing isolates were assigned to "high-risk clones", ST111 and ST235, while higher genetic diversity was observed in the group of non-carbapenemase-producing isolates, which were assigned to 11 different clones. Three main types of class 1 integrons carrying $b l a_{\mathrm{VIM}}$-like genes were found. The VIM-2-encoding integron In59, which was firstly described in France in 1998 [21], was the most common integron type identified in P. aeruginosa of STs 111, 235 and 773. An In59-like integron was also the predominant type among ST111 P. aeruginosa isolates collected from UK hospital laboratories [36]. Additionally, the VIM-4-encoding integron In595 was identified in 11 ST235 P. aeruginosa, while the integron In1760, expressing VIM-2 MBL, was found among ST395 isolates.

In $P$. aeruginosa isolates analyzed with Illumina sequencing, WGS data confirmed the chromosomal location of VIM-encoding integrons. This finding is in agreement with the clonal spread of VIM-producing pathogens in our setting. Of note, a novel genomic island (designated as PAGI-8709) associated with the bla $a_{\mathrm{VIM}-2}$-carrying integron In1760 was characterized from ST395 isolates. Additionally, WGS data confirmed the absence of carbapenemase-encoding genes in nine carbapenem-resistant $P$. aeruginosa that were positive in the EDTA-meropenem test. However, other traits corresponding to mutations in $\operatorname{mexR}, n f x B$, and $\operatorname{mex} T$ genes, which regulate the 
MexAB-OprM, MexCD-OprJ, and MexEF-OprN multidrug efflux systems, and $\operatorname{oprD}$ were found in non-carbapenemase-producing isolates.

Furthermore, analysis of WGS data demonstrated that "high-risk clones", ST111 and ST235, contributing to the spread of VIM-type carbapenemases in UHL exhibited a vast variety in their armamentariums, including resistance genes, virulence genes and/or a CRISPR/Cas system. Those features could be involved in the establishment of ST111 and ST235 isolates into hospital environments. The presence of a CRISCPR/Cas system was identified in carbapenemase-producing isolates, belonging to STs 235 and 395. Interestingly, both ST395 isolates exhibited identical type I-E CRISPR/Cas systems, further indicating their clonal relationship. A similar observation was also made for all ST235 VIM-4-producing isolates, which carried type I-C CRISPR/Cas systems. These findings restated that CRISPR/Cas systems can be used as tools for typing of bacteria. Bacteria harboring an active CRISPR/Cas system present a selective advantage against the income of harmful DNA [37]. However, this inhibition may have a negative impact on cases where horizontal gene transfer would benefit bacteria by making them more resistant. Recently, an additional function has been attributed to the CRISPR/Cas system in the regulation of genes associated with virulence [38].

\section{Conclusions}

In conclusion, this is the first study analyzing the presence of CRISPR/Cas systems in carbapenemase-producing P. aeruginosa isolates of Greek origin. In line with previous studies [34,35], our data confirmed the presence of type I-C, I-E and I-F systems in P. aeruginosa of clinical origin, and their strong association with specific lineages. Isolates, which belonged to the same clone, exhibited identical CRISPR/Cas systems underlined the fact that CRISPR arrays is a simple, but precise, genotyping tool that can be used to track pathogenic bacterium. Additionally, our findings that are consistent with the results of previous studies $[13,20,39]$ highlighted the important role of high-risk clones, namely, STs 111 and 235, in the successful dissemination of clinically important resistance determinants. Isolates, belonging to STs 111 and 235 harbored a vast variety in their armamentariums, including resistance genes, virulence genes and/or a CRISPR/Cas system, which could be involved in their successful dissemination. Therefore, recognition of carbapenemase-producing P. aeruginosa hyper-epidemic clones by molecular tools, and more specifically of WGS [40], represents an important step towards tracing transmission routes, developing targeted control and prevention strategies, and monitoring their effectiveness.

Author Contributions: C.C.P., A.V. and V.G. carried out experimental work. A.V., V.G., S.X., I.B. and J.H. assisted in the obtention of data. C.C.P. and E.P. played an important role in interpreting the results and writing the manuscript. C.C.P. supervised the experiments and revised the final manuscript. All authors have read and agreed to the published version of the manuscript.

Funding: This work was funded by the Research Committee of the University of Thessaly. It was also funded in part by the project Nr. CZ.02.1.01/0.0/0.0/16_019/0000787 “Fighting Infectious Diseases" provided by the Ministry of Education Youth and Sports of the Czech Republic.

Acknowledgments: We thank Katerina Tsilipounidaki for technical assistance.

Conflicts of Interest: The authors declare no conflict of interest.

\section{References}

1. Nicolle, L.E. Complicated urinary tract infection in adults. Can. J. Infect. Dis. Med. 2005, 16, 349-360. [CrossRef] [PubMed]

2. WHO. Global Priority List of Antibiotic-Resistant Bacteria to Guide Research, Discovery, and Development of New Antibiotics. 2017. Available online: http://www.who.int/medicines/publications/global-prioritylistantibiotic-resistant-bacteria/en/ (accessed on 27 February 2020).

3. Livermore, D.M. Multiple mechanisms of antimicrobial resistance in Pseudomonas aeruginosa: Our worst nightmare? Clin. Infect. Dis. 2002, 34, 634-640. [CrossRef] [PubMed] 
4. Tzouvelekis, L.S.; Markogiannakis, A.; Psichogiou, M.; Tassios, P.T.; Daikos, G.L. Carbapenemases in Klebsiella pneumoniae and other Enterobacteriaceae: An evolving crisis of global dimensions. Clin. Microbiol. Rev. 2012, 25, 682-707. [CrossRef] [PubMed]

5. Tsakris, A.; Pournaras, S.; Woodford, N.; Palepou, M.F.; Babini, G.S.; Douboyas, J.; Livermore, D.M. Outbreak of infections caused by Pseudomonas aeruginosa producing VIM-1 carbapenemase in Greece. J. Clin. Microbiol. 2000, 38, 1290-1292. [CrossRef] [PubMed]

6. Giakkoupi, P.; Petrikkos, G.; Tzouvelekis, L.S.; Tsonas, S.; Legakis, N.J.; Vatopoulos, A.C.; WHONET Greece Study Group. Spread of integron-associated VIM-type metallo-beta-lactamase genes among imipenem-nonsusceptible Pseudomonas aeruginosa strains in Greek hospitals. J. Clin. Microbiol. 2003, 41, 822-825. [CrossRef] [PubMed]

7. Pournaras, S.; Tsakris, A.; Maniati, M.; Tzouvelekis, L.S.; Maniatis, A.N. Novel variant (bla(VIM-4)) of the metallo-beta-lactamase gene bla(VIM-1) in a clinical strain of Pseudomonas aeruginosa. Antimicrob. Agents Chemother. 2002, 46, 4026-4028. [CrossRef]

8. Pournaras, S.; Maniati, M.; Petinaki, E.; Tzouvelekis, L.S.; Tsakris, A.; Legakis, N.J.; Maniatis, A.N. Hospital outbreak of multiple clones of Pseudomonas aeruginosa carrying the unrelated metallo-beta-lactamase gene variants bla $a_{\mathrm{VIM}-2}$ and bla $a_{\mathrm{VIM}-4}$. J. Antimicrob. Chemother. 2003, 51, 1409-1414. [CrossRef]

9. Liakopoulos, A.; Mavroidi, A.; Katsifas, E.A.; Theodosiou, A.; Karagouni, A.D.; Miriagou, V.; Petinaki, E. Carbapenemase-producing Pseudomonas aeruginosa from Central Greece: Molecular epidemiology and genetic analysis of class I integrons. BMC Infect. Dis. 2013, 13, 505. [CrossRef]

10. EUCAST. European Committee on Antimicrobial Susceptibility Testing (EUCAST) of the European Society of Clinical Microbiology and Infectious Diseases (ESCMID). Determination of minimum inhibitory concentrations (MICs) of antibacterial agents by broth dilution. Clin. Microbiol. Infect. 2003, 9, ix-xv. [CrossRef]

11. Wayne, P.A. Clinical and Laboratory Standards Institute. Performance Standards for Antimicrobial Susceptibility Testing; CLSI 2013, 23rd International Supplement, CLSI document M100-S23; Clinical and Laboratory Standards Institute: Wayne, PA, USA, 2013.

12. Lee, K.; Lim, Y.S.; Yong, D.; Yum, J.H.; Chong, Y. Evaluation of the Hodge test and the imipenem-EDTA double-disk synergy test for differentiating metallo-b-lactamase-producing isolates of Pseudomonas spp. and Acinetobacter spp. J. Clin. Microbiol. 2003, 41, 4623-4629. [CrossRef]

13. Papagiannitsis, C.C.; Medvecky, M.; Chudejova, K.; Skalova, A.; Rotova, V.; Spanelova, P.; Jakubu, V.; Zemlickova, H.; Hrabak, J. Molecular Characterization of carbapenemase-producing Pseudomonas aeruginosa of Czech origin and evidence for clonal spread of extensively resistant Sequence Type 357 expressing IMP-7 metallo- $\beta$-lactamase. Antimicrob. Agents Chemother. 2017, 61, e01811-17. [CrossRef] [PubMed]

14. Curran, B.; Jonas, D.; Grundmann, H.; Pitt, T.; Dowson, C.G. Development of a multilocus sequence typing scheme for the opportunistic pathogen Pseudomonas aeruginosa. J. Clin. Microbiol. 2004, 42, 5644-5649. [CrossRef] [PubMed]

15. Moura, A.; Soares, M.; Pereira, C.; Leitão, N.; Henriques, I.; Correia, A. INTEGRALL: A database and search engine for integrons, integrases and gene cassettes. Bioinformatics 2009, 25, 1096-1098. [CrossRef] [PubMed]

16. Barton, B.M.; Harding, G.P.; Zuccarelli, A.J. A general method for detecting and sizing large plasmids. Anal. Biochem. 1995, 226, 235-240. [CrossRef] [PubMed]

17. Zankari, E.; Hasman, H.; Cosentino, S.; Vestergaard, M.; Rasmussen, S.; Lund, O.; Aarestrup, F.M.; Larsen, M.V. Identification of acquired antimicrobial resistance genes. J. Antimicrob. Chemother. 2012, 67, 2640-2644. [CrossRef]

18. Liu, B.; Zheng, D.D.; Jin, Q.; Chen, L.H.; Yang, J. VFDB 2019: A comparative pathogenomic platform with an interactive web interface. Nucleic Acids Res. 2019, 47, D687-D692. [CrossRef]

19. Couvin, D.; Bernheim, A.; Toffano-Nioche, C.; Touchon, M.; Michalik, J.; Neron, B.; Rocha, E.P.C.; Vergnaud, G.; Gautheret, D.; Pourcel, C. CRISPRCasFinder, an update of CRISRFinder, includes a portable version, enhanced performance and integrates search for Cas proteins. Nucleic Acids Res. 2018, 46, W246-W251. [CrossRef]

20. Woodford, N.; Turton, J.F.; Livermore, D.M. Multiresistant Gram-negative bacteria: The role of high-risk clones in the dissemination of antibiotic resistance. FEMS Microbiol. Rev. 2011, 35, 736-755. [CrossRef] 
21. Poirel, L.; Lambert, T.; Turkoglu, S.; Ronco, E.; Gaillard, J.L.; Nordmann, P. Characterization of class 1 integrons from Pseudomonas aeruginosa that contain the bla $a_{\mathrm{VIM}-2}$ carbapenem-hydrolyzing b-lactamase gene and of two novel aminoglycoside resistance gene cassettes. Antimicrob. Agents Chemother. 2001, 45, 546-552. [CrossRef]

22. Samuelsen, Ø.; Toleman, M.A.; Sundsfjord, A.; Rydberg, J.; Leegaard, T.M.; Walder, M.; Lia, A.; Ranheim, T.E.; Rajendra, Y.; Hermansen, N.O.; et al. Molecular epidemiology of metallo- $\beta$-lactamase-producing Pseudomonas aeruginosa isolates from Norway and Sweden shows import of international clones and local clonal expansion. Antimicrob. Agents Chemother. 2010, 54, 346-352. [CrossRef]

23. Yano, H.; Genka, H.; Ohtsubo, Y.; Nagata, Y.; Top, E.M.; Tsuda, M. Cointegrate-resolution of toluene-catabolic transposon Tn4651: Determination of crossover site and the segment required for full resolution activity. Plasmid 2013, 69, 24-35. [CrossRef] [PubMed]

24. Breidenstein, E.B.M.; de la Fuente-Nunez, C.; Hancock, R.E.W. Pseudomonas aeruginosa: All roads lead to resistance. Trends Microbiol. 2011, 19, 419-426. [CrossRef] [PubMed]

25. Jalal, S.; Wretlind, B. Mechanisms of quinolone resistance in clinical strains of Pseudomonas aeruginosa. Microb. Drug Resist. 1998, 4, 257-261. [CrossRef] [PubMed]

26. Walsh, F.; Amyes, S.G. Carbapenem resistance in clinical isolates of Pseudomonas aeruginosa. J. Chemother. 2007, 19, 376-381. [CrossRef]

27. Lanotte, P.; Watt, S.; Mereghetti, L.; Dartiguelongue, N.; Rastegar-Lari, A.; Goudeau, A.; Quentin, R. Genetic features of Pseudomonas aeruginosa isolates from cystic fibrosis patients compared with those of isolates from other origins. J. Med. Microbiol. 2004, 53, 73-81. [CrossRef]

28. Azimi, S.; Kafil, H.S.; Baghi, H.B.; Shokrian, S.; Najaf, K.; Asgharzadeh, M.; Yousefi, M.; Shahrivar, F.; Aghazadeh, M. Presence of exoY, exoS, exou and exoT genes, antibiotic resistance and biofilm production among Pseudomonas aeruginosa isolates in Northwest Iran. GMS Hyg. Infect. Control. 2016, 11, Doc04. [CrossRef]

29. Roy-Burman, A.; Savel, R.H.; Racine, S.; Revadigar, N.S.; Fujimoto, J.; Sawa, T.; Frank, D.W.; Wiener-Kronish, J.P. Type III protein secretion is associated with death in lower respiratory and systemic Pseudomonas aeruginosa infections. J. Infect. Dis. 2001, 183, 1767-1774. [CrossRef]

30. Finck-Barbancon, V.; Goranson, J.; Zhu, L.; Wiener-Kronish, J.P.; Fleiszig, S.M.; Wu, C.; Mende-Mueller, L.; Frank, D.W. ExoU expression by Pseudomonas aeruginosa correlates with acute cytotoxicity and epithelial injury. Mol. Microbiol. 1997, 25, 547-557. [CrossRef]

31. Van't Wout, E.F.; Van Schadewijk, A.; Van Boxtel, R.; Dalton, L.E.; Clarke, H.J.; Tommassen, J.; Marciniak, S.J.; Hiemstra, P.S. Virulence factors of Pseudomonas aeruginosa induce both the unfolded protein and integrated stress responses in airway epithelial cells. PLoS Pathog. 2015, 11, e1004946. [CrossRef]

32. Jamunadevi, S.; Balashanmugam, P.; Muralitharan, G.; Kalaichelvan, P.T. Molecular characterization of pathogenic and non-pathogenic Pseudomonas aeruginosa with special reference to phenazine gene. J. Mod. Biotechnol. 2012, 1, 70-74.

33. Karah, N.; Samuelsen, O.; Zarrilli, R.; Sahl, J.W.; Wai, S.N.; Uhlin, B.E. CRISPR-cas subtype I-Fb in Acinetobacter baumannii: Evolution and utilization for strain subtyping. PLoS ONE 2015, 10, e0118205. [CrossRef]

34. van Belkum, A.; Soriaga, L.B.; LaFave, M.C.; Akella, S.; Veyrieras, J.B.; Barbu, E.M.; Shortridge, D.; Blanc, B.; Hannum, G.; Zambardi, G.; et al. Phylogenetic distribution of CRISPR-Cas systems in antibiotic-resistant Pseudomonas aeruginosa. mBio 2015, 6, e01796-15. [CrossRef] [PubMed]

35. de Oliveira Luz, A.C.; da Silva, J.M.A.; Rezende, A.M.; de Barros, M.P.S.; Leal-Balbino, T.C. Analysis of direct repeats and spacers of CRISPR/Cas systems type I-F in Brazilian clinical strains of Pseudomonas Aeruginosa. Mol. Genet. Genom. 2019, 294, 1095-1105. [CrossRef]

36. Wright, L.L.; Turton, J.F.; Hopkins, K.L.; Livermore, D.M.; Woodford, N. Genetic environment of metallo- $\beta$-lactamase genes in Pseudomonas aeruginosa isolates from the UK. J. Antimicrob. Chemother. 2015, 70, 3250-3258. [CrossRef] [PubMed]

37. Ostria-Hernandez, M.L.; Sánchez-Vallejo, C.J.; Ibarra, J.A.; Castro-Escarpulli, G. Survey of clustered regularly interspaced short palindromic repeats and their associated Cas proteins (CRISPR/Cas) systems in multiple sequenced strains of Klebsiella pneumoniae. BMC Res. Notes 2015, 8, 332. [CrossRef]

38. Hatoum-Aslan, A.; Marraffini, L.A. Impact of CRISPR immunity on the emergence and virulence of bacterial pathogens. Curr. Opin. Microbiol. 2014, 17, 82-90. [CrossRef] 
39. Edelstein, M.V.; Skleenova, E.N.; Shevchenko, O.V.; D’souza, J.W.; Tapalski, D.V.; Azizov, I.S.; Sukhorukova, M.V.; Pavlukov, R.A.; Kozlov, R.S.; Toleman, M.A.; et al. Spread of extensively resistant VIM-2-positive ST235 Pseudomonas aeruginosa in Belarus, Kazakhstan, and Russia: A longitudinal epidemiological and clinical study. Lancet Infect. Dis. 2013, 13, 867-876. [CrossRef]

40. Hrabak, J.; Bitar, I.; Papagiannitsis, C.C. Combination of mass spectrometry and DNA sequencing for detection of antibiotic resistance in diagnostic laboratories. Folia Microbiol. 2020, 65, 233-243. [CrossRef]

Publisher's Note: MDPI stays neutral with regard to jurisdictional claims in published maps and institutional affiliations.

(C) 2020 by the authors. Licensee MDPI, Basel, Switzerland. This article is an open access article distributed under the terms and conditions of the Creative Commons Attribution (CC BY) license (http://creativecommons.org/licenses/by/4.0/). 